MATEC Web of Conferences 25,02017

DOI: $10.1051 /$ matec conf/ 20152502017

(2015)

(C) Owned by the authors, published by EDP Sciences, 2015

\title{
Research on Preparation and Properties of Coated Polyether Silicone Microencapsulation Based on Polystyrene
}

\author{
Shuiyun Ren, Huangjun Huang, Jianwei Hu \& Hongjing Wan \\ Department of Ministry of Science, Ordnance Engineering College, Shijiazhuang, Hebei, China
}

\begin{abstract}
By the method of interfacial polymerization, the novel microcapsules were prepared with polystyrene as wall material and polyether silicone as core material. In order to demonstrate the morphologies, particle size distribution and properties of microcapsules, scanning electron microscopy (SEM), laser particle size analysis and infrared spectroscopy (FTIR) are also applied in the experiments, and the thermal stability of microcapsules is obtained by thermal gravimetric (TG) and differential scanning calorimetry (DSC). The material ratio (core to wall in weight) of microcapsules and stirring rate ratio are discussed. The results show that under the optimum process conditions of a material ratio (core to wall in weight) which is $4: 10$ and agitating rate of $500 \mathrm{r} \cdot \mathrm{min}^{-1}$, the synthesized polyether silicone microcapsules have a higher yield and the coating and densification properties; their average size is $210 \mu \mathrm{m}$, and the thermal stability temperature can reach up to $390^{\circ} \mathrm{C}$.
\end{abstract}

Keywords: microcapsules; polyether silicone; polystyrene; interfacial polymerization; thermal stability

\section{INTRODUCTION}

Silicone oil has an extremely wide range of applications such as the key areas of cosmetics, hair products and machinery manufacturing ${ }^{[1]}$. The introduction of polyether group of the polyether silicone provides good wettability, low surface tension and physiologically inert, and its products have advantages such as better moisture absorption, antistativity and cleaning easiness and so on ${ }^{[2]}$. It is rarely reported about the microencapsulation of silicone oil, especially the microencapsulation of polyether silicone oil, which has never been reported. Pang Hongbo successfully prepared silica shells of silicone oil microcapsules with the fine emulsion technology. The microcapsules of this silicone oil emulsion are mixed with an aqueous acrylic to formulate into anti-icing coating, which can reduce long-term performance ${ }^{[3]}$. As the polyurea formaldehyde is regarded as capsule material and the vinyl silicone oil as core, Ai Qiushi, MINORITIES, who adopts the method of a "two-step" and "one-step", preparing a kind of self-repair microcapsules of vinyl silicone oil by in situ polymerization ${ }^{[4]}$. Polystyrene is commonly used as the capsule material of polymer composite materials; microcapsules with the polystyrene-coated method have advantages such as the good cystic and dense sound, the uniform particle size and so on ${ }^{[5,6]}$.

In this paper, styrene-divinylbenzene as capsule material, polyether silicone oil as core and the uniform particle size of polystyrene-coated polyether silicone oil microcapsules are prepared successfully in using the method of interfacial polymerization for the first time. The surface morphology of the microcapsules, the particle size characterization and the thermal sta- bility are respectively characterized by scanning electron microscopy and laser particle size analysis, infrared spectroscopy and DTA-TGA. It discusses how the capsule core ratio and the stirring rate affect the synthesis of polyether silicone oil microcapsules.

\section{EXPERIMENT}

\subsection{Reagents}

Gum arabic powder: AR, Tianjin Bodie Chemical Corporation; ethanol, potassium persulfate: AR, Tianjin Yong-largest chemical reagents Limited; divinyl benzene, styrene: AR, Tianjin Guangfu Fine Chemical Institute; benzoyl peroxide: AR, Tianjin Chemical Reagent Factory $\mathrm{Fu}$ Chen; polyether silicone oil (L580): industrial, Shijiazhuang blue Technology Limited; epoxy reactive diluent 501 \#: AR, Hong Kong Hui Pharmaceutical Group

\subsection{Main instruments}

IR: nicolet6700 Fourier transform infrared spectroscopy of US ThermoFisher company; scanning electron microscope: S-4800-1 field emission scanning electron microscope of Japan HITACHI company; laser particle size analyzer: LS900 laser particle size analyzer of China, Europe and the United States grams company; TGA: STD-2960 DTA - TGA coupled with a thermal analyzer of American TA company; heating stirrer: DF-101S collector-type temperature magnetic heating stirrer of China Kunshan Sunlord Electronics Co. 


\section{MATEC Web of Conferences}

\subsection{Preparation of polyether silicone-coated poly- styrene microcapsules}

A certain mass fraction of gum arabic solution is prepared with distilled water, and a certain quality of benzoyl peroxide, divinylbenzene (the ratio of styrene and divinylbenzene is 12.5:1) and poly ether silicone oil are dissolved in the epoxy reactive diluent styrene, then these two solutions are added into the three-necked flask and heated to $35^{\circ} \mathrm{C}$, stirring for $0.5 \mathrm{~h}$ respectively. Finally, the $\mathrm{O} / \mathrm{W}$ type emulsion is formed in the nitrogen atmosphere which replaces the atmosphere in the flask. It needs to be slowly heated to $70-80^{\circ} \mathrm{C}$ and stirred for $5 \mathrm{~h}$ at the speed of $400 \mathrm{r} / \mathrm{min}$ for continuous reaction, then a small amount of potassium persulfate solution (mass fraction of $1.0 \%$ ) is added to continue the reaction for $3 \mathrm{~h}$; samples are filtrated to separate and, they are washed with distilled water and anhydrous ethanol after the completed reaction; finally, the polystyrene-coated polyether silicone after microcapsule samples are obtained through drying under vacuum.

\subsection{Performance testing}

(1) FT-IR test: take a certain amount of microcapsules to ground, and then prepare the potassium bromide tablet for testing.

(2) Morphology analysis: carry out spraying process and observe the surface morphology of microcapsules by scanning electron microscope.

(3)Verify the capsule core structure: dispose a certain amount of polyether silicone oil microcapsules to the two clean glasses and apply an external force to observe whether extrusion of the oil liquid (glass sheet extrusion method).

(4)Particle size analysis: aqueous dispersion, measuring size distribution of sample microcapsule by a laser particle size analyzer.

(5) Thermal gravimetric analysis: heating at the rate of $10^{\circ} \mathrm{C} / \mathrm{min}$ under the nitrogen atmosphere and conduct TGA/DSC analysis simultaneously.

\section{RESULTS AND DISCUSSION}

\subsection{FT-IR test and analysis}

The curve $a$ is the infrared spectrum of polyether silicone; the curve $\mathrm{b}$ is the styrene infrared spectrum; the curve $\mathrm{c}$ is the infrared spectrum of the polystyrene-coated silicone polyether in Figures 1 and 2. It can be seen from the curve a that the absorption peak at $1,260 \mathrm{~cm}^{-1}$ is $\mathrm{Si}-\mathrm{CH}_{3}$ symmetric bending; the absorption peak at $1,000-1,100 \mathrm{~cm}^{-1}$ is the absorption of $\mathrm{C}-\mathrm{O}-\mathrm{C}, \mathrm{C}-\mathrm{O}$ and $\mathrm{Si}-\mathrm{O}-\mathrm{Si}$; the peak at $760-825 \mathrm{~cm}^{-1}$ is the absorption peaks of an epoxy group and a silicone resulting from generation, indicating that the described silicone polyether has a structure with a hydroxyl group, an amino group, an ether group and a silicone. $539 \mathrm{~cm}^{-1}, 694 \mathrm{~cm}^{-1}, 1,450 \mathrm{~cm}^{-1}$ and $1,600 \mathrm{~cm}^{-1}$ are re- spectively the absorption peak of benzene styrene in curve $b$. In curve $c$, there are characteristic peaks of polyether silicone and styrene, namely, they are characteristic absorption peaks of polystyrene amorphous body at $835 \mathrm{~cm}^{-1}$, providing that the polymerization of styrene coated the polyether silicone successfully.

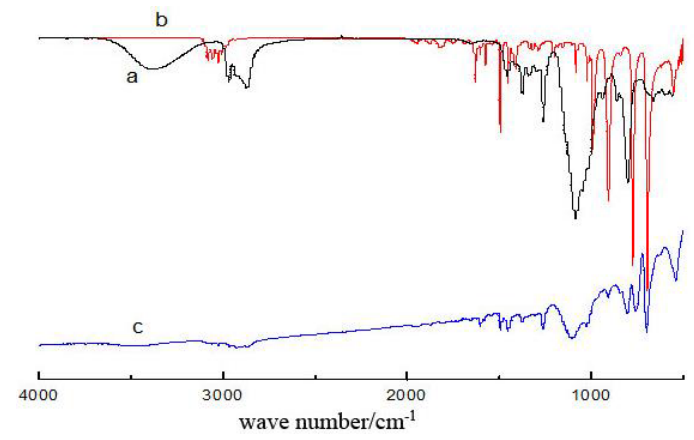

Figure 1. Polyether silicone oil (a), styrene (b) and a microcapsule (c)

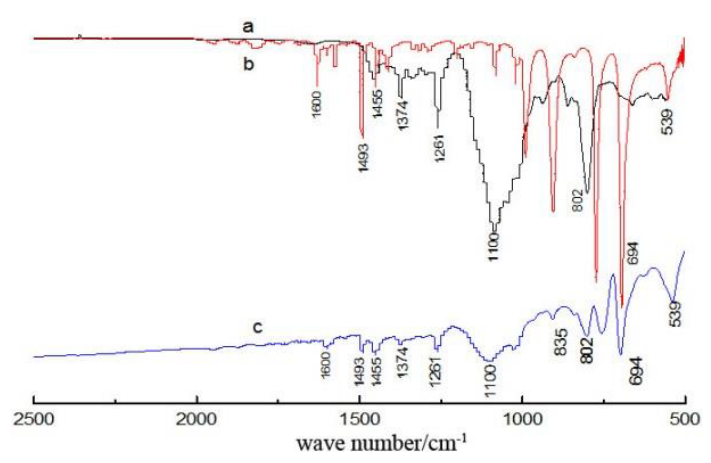

Figure 2. IR spectrum of polyether silicone oil (a), styrene (b) and a microcapsule (c)

\subsection{Verify the capsule core structure}

Verify capsule core structure: weigh a small amount of silicone polyether microcapsule samples to two sheets of a piece of clean glass, provide the existence of oily phase in the sample microcapsule by squeezing and observe the oil phase overflowing ${ }^{[7]}$.

It is the completion of microcapsule sample (a) which is after placing for $7 \times 24$ hours (b) in Figure 3. From Figure 2 (a), it can be seen that coating of polyether silicone oil is good in the microcapsule. From Figure 2 (b), it shows the shell structure of microcapsules rupture sample. Thus it proves the polyether silicone-coated polystyrene successfully.

\subsection{Determine proportions of capsule material and the core material}

The proportion of the microcapsule wall material and 
the capsule core microcapsules mainly affects the thickness and surface morphology ${ }^{[8,9]}$. To find a suitable capsule core ratio, three experiments are respectively conducted with the capsule core ratio of $3: 10$, 4:10 and 5:10, and SEM is shown in Figure 3:

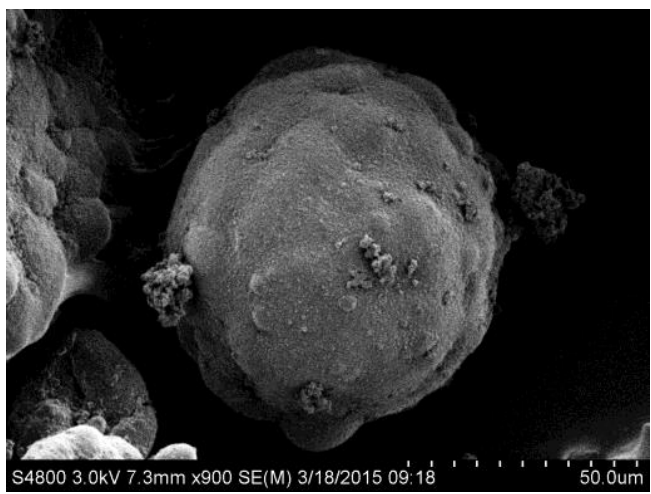

(a) Completion of microcapsule sample

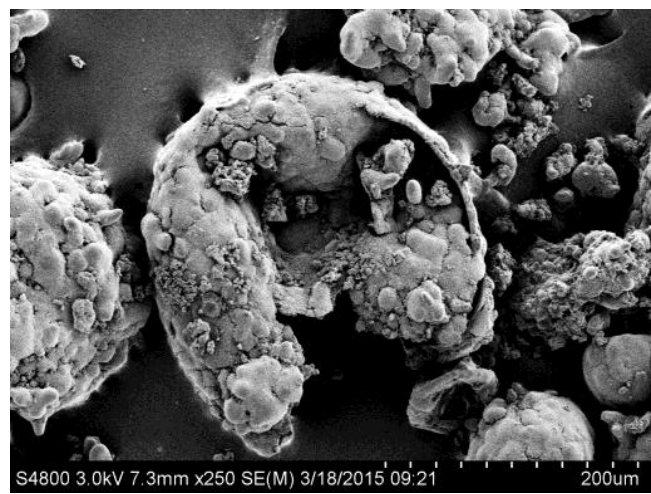

(b) The microcapsule sample after placing for $7 \times 24$ hours

Figure 3. Scanning electron micrographs of polyether silicone oil microcapsules

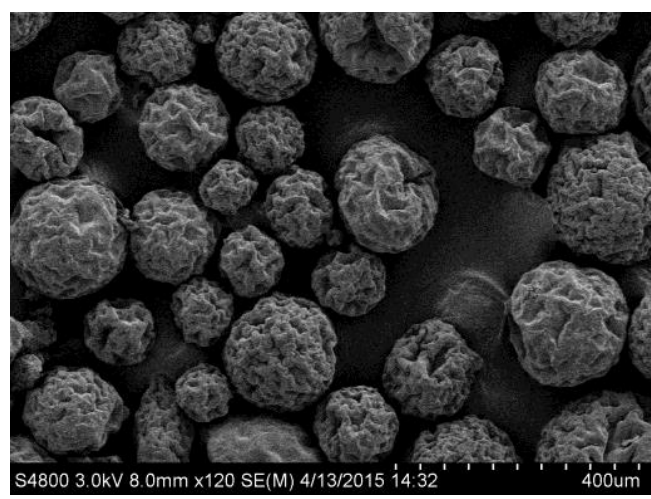

(a) Capsule core ratio which is 3:10

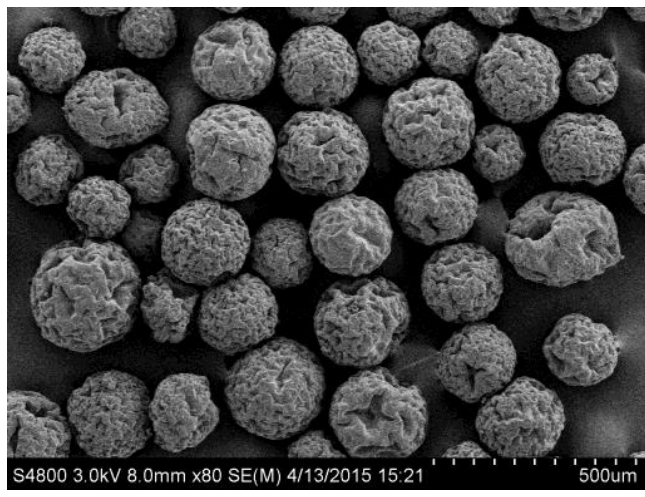

(b) Capsule core ratio which is $4: 10$

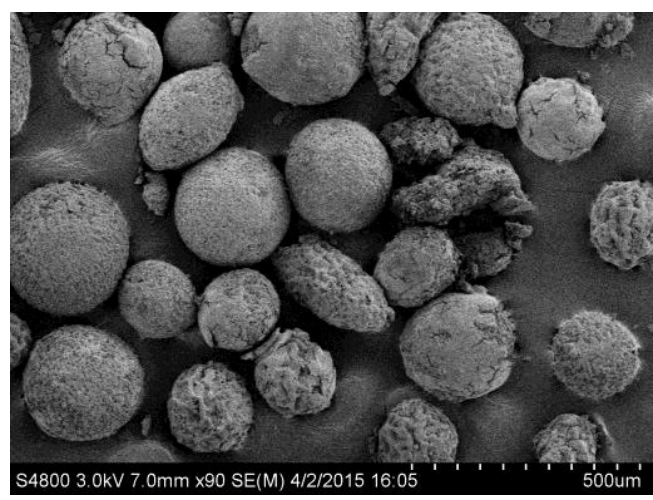

(c) Capsule core ratio which is $5: 10$

Figure 4. SEM of polyether silicone oil microcapsules with different capsule core ratios

From Figure 4, with the capsule core ratio which is $3: 10$, it can be seen that the spheronization and compactness of microcapsules is poor and the surface topography is uneven. When the capsule core ratio is $4: 10$, the coating of microcapsules is good and the surface morphology is better. When the capsule core ratio is $5: 10$, the microcapsule wall is thick, low sac core content and even solid polystyrene spheres, which is not in favor of the further use of polyether silicone oil. Thus, the optimal ratio of capsule core is $4: 10$.

\subsection{Determination of stirring rate}

The stirring rate is the most important factor to affect the microcapsule particle size ${ }^{[10]}$, and it is used in this paper to study the effect of different stirring rate of $400-600 \mathrm{r} / \mathrm{min}$ on the particle size of polyether silicone microcapsules. It shows the SEM photograph of microcapsule samples with different stirring rates in Figure 5: 


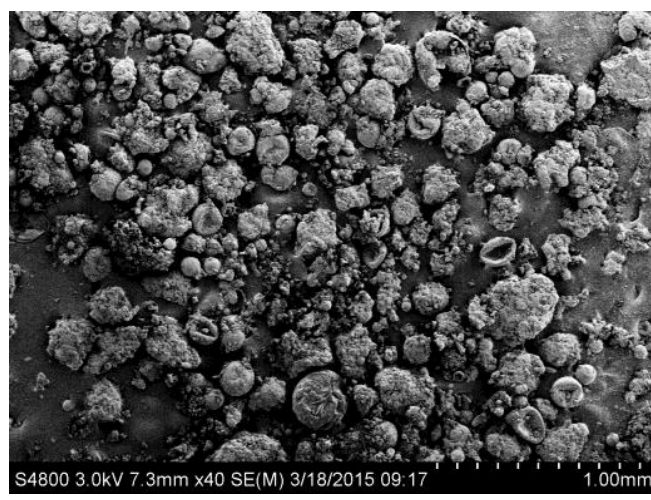

(a) $400 \mathrm{r} / \mathrm{min}$

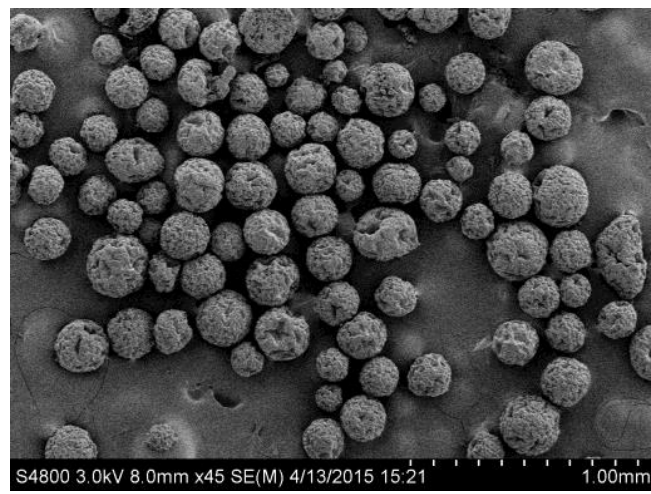

(b) $500 \mathrm{r} / \mathrm{min}$

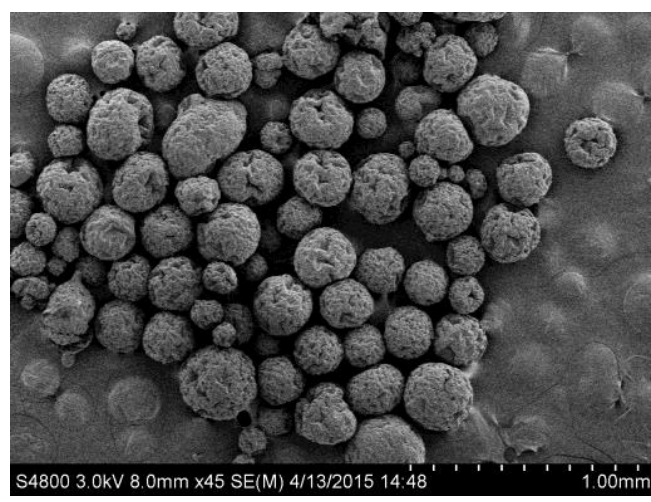

(c) $600 \mathrm{r} / \mathrm{min}$

Figure 5. Scanning electron micrograph of the microcapsules sample under different stirring rates

Table 1. Effect of stirring rate on the microcapsule synthesis

\begin{tabular}{llll}
\hline \multirow{2}{*}{ Project } & \multicolumn{3}{l}{ Stirring rate $(\mathrm{r} / \mathrm{min})$} \\
\cline { 2 - 4 } & 400 & 500 & 600 \\
\hline cladding & poor & good & good \\
the average particle size & 245 & 210 & 275 \\
distribution of dispersion & 4.21 & 2.16 & 3.42 \\
\hline
\end{tabular}

It can be seen from Figure 4 and Table 1 that microcapsules' cladding is poor, the average particle size is largely and widely distributed, and some are reunit- ed with the lower stirring rate. With the increase of stirring rate, the microcapsules' average particle size is decreased, and the distribution becomes narrower, which reduces the effect of reunion. Microcapsules' particle size is uniform and widely distributed when the stirring rate is higher. The reason is that as the shearing force is increased, the capsule core droplet size becomes smaller, and the synthetic microcapsule particle size becomes small, and the smooth surface is conducive to forming when the stirring rate is increased ${ }^{[11,12]}$. When the stirring rate continues to increase, the stability of the capsule core droplets is undermined, resulting in widened size distribution of microcapsule. It shows that the stirring rate which is $500 \mathrm{r} / \mathrm{min}$ is more appropriate in the experiment.

\subsection{Thermal stability of microcapsules}

The microcapsules are dried in vacuum for 6 hours at $40^{\circ} \mathrm{C}$, simultaneously, TGA/DSC analysis is conducted to test the thermal vector quality (TGA) curve and the DSC curve of the microcapsules, and the results are shown in Figure 6. It can be seen from the TGA curves that there are two phases of microcapsules' quality thermal vector. The first one is at $100-320^{\circ} \mathrm{C}$ where the vector weight is $12 \%$, and it can be seen from the corresponding DSC curve at this stage that there is a distinct endothermic peak at $149^{\circ} \mathrm{C}$ caused by the decomposition of the silicone polyether core. The second is at the $320-430^{\circ} \mathrm{C}$ where the weight loss rate is $82 \%$, and it can be seen from the corresponding DSC curves that there is a distinct endothermic peak at $390^{\circ} \mathrm{C}$ caused by the decomposition of the capsule material polystyrene. So the results show that polyether silicone oil microcapsules have good thermal stability in the following $149^{\circ} \mathrm{C}$.

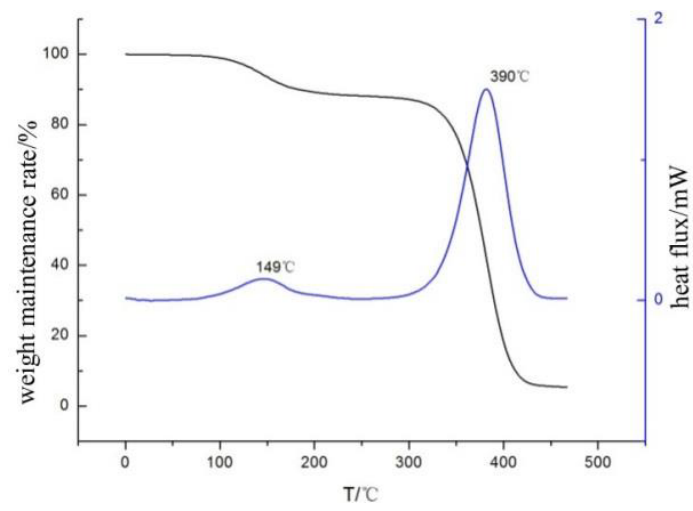

Figure 6. TGA/DSC curves of polyether silicone oil microcapsules

\section{CONCLUSIONS}

It is successful to prepare the polyether silicone-coated polystyrene microcapsules for the first time by using the styrene and divinylbenzene as the capsule and 
polyether silicone oil as core. According to the experiments and results analysis, when the capsule core ratio is $4: 10$ and the stirring rate is $400 \mathrm{r} / \mathrm{min}$, the $\mathrm{mi}$ crocapsules have a higher cladding rate and compact structure, and the average particle diameter of microcapsules is $210 \mu \mathrm{m}$, the thermal stability temperature is $149^{\circ} \mathrm{C}$, so it can be stored for a long time at room temperature. This study has made some contributions for the expanded multi-functional application on polyether silicone.

\section{ACKNOWLEDGEMENT}

This paper is financially supported by the National Natural Science Foundation of China (No. 51103178).

\section{REFERENCES}

[1] Wang Sha, Sun glory, \& Cai Xin. 2013. Application progress of silicone oil in the preparation of polymer. Colloid and Polymer, 31 (2): 86-89.

[2] Han Lulu, Biliang Wu, \& Zhao Zhendong, etc. 2013. Microcapsules of progress in application and characterization. New Chemical Materials, 41(9): 1006-3536.

[3] Pang Hongbo, ZHOU school, \& Wu Limin. 2011. Preparation of silicone oil microcapsules and the properties of aqueous anti-icing coating, Plating and Finishing, 30 (6): 57-61.
[4] Ai Qiu, Zhang Qiuyu, \& Xing Ruiying. 2010. One-step method for preparation of poly coated urea formaldehyde reactive vinyl silicone oil microcapsules. Chinese adhesives, 19(4): 13-17.

[5] Qiu Shuilai,Zhu Yulu, \& Huang Zhengqi, etc. 2014. The research and application of polymer microcapsules. New Chemical Materials, (9).

[6] Liu Y, Lü Y, \& Luo G. 2013. Preparation of microcapsule-supported Pd catalyst using a microfluidic platform. Chinese Journal of Catalysis, 34(9): 1635-1643.

[7] Song Y, Yu H, \& Jo Y, et al. 2013. Sunlight-induced Self-healing of a Microcapsule-type Protective Coating. Acs Applied Materials \& Interfaces, 5(4): 1378-1384.

[8] Zhu D Y, Rong M Z, \& Zhang M Q. 2013. Preparation and characterization of multilayered microcapsule-like microreactor for self-healing polymers. Polymer, 54(16): $4227-4236$

[9] Yang Hui, 2014. Jing preparation and performance evaluation of floating microcapsules. Modern Chemical Industry, 34 (2): 85-88.

[10] essay barrier, Yu Deshun, 2014. Leaves and other Fifi ginger essential oil micro research process. capsule of Guizhou Normal University, 30(3): 19-23.

[11]Wu fruit, Liu Wei, \& Wang Shibin, etc. 2014. Alginate / poly-arginine microcapsules drug loading and release properties. Chemical Engineering Progress, (5): 1271-1275.

[12]Mengxian Jun, Zhao Jing, \& Li Bin. 2013. Orthogonal test microencapsulation process raspberry anthocyanins. Food Science, 34(14): 173-177. 